How to reference this article Majorana, B. (2019). L'anti-cristiana. Sulle attrici professioniste della prima età moderna. Italica Wratislaviensia, 10(2), 85-101.

DOI: http://dx.doi.org/10.15804/IW.2019.10.1.18

Bernadette Majorana

Università degli Studi di Bergamo

bernadette.majorana@unibg.it

ORCID: 0000-0002-6322-7996

\title{
L'ANTI-CRISTIANA. SULLE ATTRICI PROFESSIONISTE DELLA PRIMA ETÀ MODERNA
}

\section{THE ANTI-CHRISTIAN WOMAN. ON PROFESSIONAL ACTRESSES IN THE EARLY MODERN AGE}

\begin{abstract}
The profession of the commedia dell'arte actress acquires a markedly visible position in the broad public horizon of Modern-Age Italy. The actress, in that context, places herself both at the borders of the male amateur theatre practices and amongst the virtuous feminine models (nuns, wives, virgins), conflicting with both. These counter-positions must have been very visible, particularly to the spectators, who could easily draw comparisons both with the amateur shows, where only male actors played, and with different kinds of feminine characters. These contrasts are explored in this article using moral essays of the time, including the equivalence between actress and prostitute put forward by a lengthy treatise in 1646 by G.D. Ottonelli.
\end{abstract}

Keywords: commedia dell'arte, actresses, amateur actors, feminine models, prostitute 
Son di Francesco consorte e compagna, con lui divido famiglia e mestiere e arte e vita, lui mi fu garante di un destino che non fosse soltanto quello di moglie e madre, o monaca o prostituta. Io fui comica $[\ldots]$.

(Bucci, Sgrosso, 2004, p. 178)

1. Le attrici della Commedia dell'Arte sono determinanti nella storia di questa forma di teatro (Ferrone, 2014, pp. 40-61, 145-147) e sono anche una specola per comprendere il conflitto che nell'Italia cattolica contrappone comici e moralisti e che riguardo alle attrici si manifesta in modo preminente (Majorana, 1996) ${ }^{1}$. Come è noto, la Commedia dell'Arte è il contesto nel quale, superata da poco la metà del Cinquecento, per la prima volta nella storia europea la donna compare in scena in maniera sistematica, come recitante e come figura e tipo drammaturgicamente centrali, nonché come professionista dotata di tecniche recitative sue proprie, nel contesto di compagnie miste. La sua comparsa suscita straordinario interesse, da un canto, e reazioni fortemente critiche, dall'altro.

Alcuni aspetti di questa storia si possono osservare tenendo conto di due questioni inevitabili, in età moderna, su cui proporrò qualche riflessione. La donna che recita, infatti, acquista rilievo e immediato successo per la novità e soprattutto per la specificità della sua presenza; ma per comprendere, allo stesso tempo, quel che si può definire il trauma sociale prodotto dal suo avvento, tale presenza può essere osservata nel suo collegarsi a due assi di riferimento: quello della recitazione maschile dei dilettanti e quello dei principali stati di vita femminili.

Si sa come dalla polemica sorta intorno alla Commedia dell'Arte emergano, in primo luogo, accuse generali contro il teatro, che riattizzano le antiche e mai sopite questioni teologico-morali sul commercio del corpo, sull'intrattenimento, la finzione, la diabolicità, l'oscenità della presenza esibita e tradotta in attrazione comica, in divertimento. In

${ }^{1}$ Si veda, nel presente volume, il contributo di Teresa Megale. 
questo quadro, l'elemento anti-femminile è centrale: si contestano l'evidenza seduttiva dell'attrice dell'Arte, la libertà di lavoro e guadagno, lo stabilire con i compagni di mestiere rapporti paritetici, il mostrarsi munita di competenze specialistiche. Tutto ciò corrisponde alla consistenza del problema così come i detrattori conflittualmente lo pongono e i comici, per contro, lo rigettano ${ }^{2}$, e riflette le ragioni teatrali e sociali che fanno di quella dell'attrice una questione contraddittoria, divisa tra fortuna e riprovazione.

Riguardo al trauma e al piacere prodotti dall'avvento delle donne sulle scene italiane, va sottolineata la prima e fondamentale condizione a cui si è accennato: il fatto, cioè, che l'attrice di mestiere compaia e si affermi in un quadro teatrale di segno opposto, dominato dalla scena maschile. La scena esclusivamente maschile di ambito dilettante (Majorana, 2000, 2013; Mazzoni, 2000; Ciancarelli, 2008) è la sola attività teatrale che in Italia, nel periodo di cui parliamo e oltre, sia alternativa a quella dei comici professionisti. Precede lo sviluppo della recitazione di mestiere, nella combinazione maschile-femminile propria dell'Arte, e perdura lungo tutto il suo evolversi. Sono due esperienze teatrali opposte e parallele, che hanno però in comune almeno un fattore, cruciale: il pubblico. Nella loro varietà indifferenziata, gli spettatori dell'una possono essere anche spettatori dell'altra.

Non si può trascurare di ritenere che per un pubblico abituato a vedere, sulle scene dei teatri, uomini soltanto e soltanto figure femminili impersonate da uomini, la comparsa di vere donne abbia costituito un fatto sconvolgente, sia sotto il profilo del passaggio dalla pura convenzione interpretativa alla mimesi recitativa imposta dal corpo, dalla voce, dal volto delle donne (l'Innamorata, ruolo clou delle attrici, diversamente dagli altri ruoli dell'Arte, e tolto l'Innamorato, non porta maschera); sia per l'incrinarsi di un intero universo morale, che aveva legittimato, in prospettiva tanto religiosa quanto laica, unicamente l'azione scenica pubblica da parte degli uomini, fenomeno imponente e unitario, oltre che da secoli ininterrotto, mediante il quale - appunto in virtù della

2 Restano fondamentali le raccolte di testi proposte da Taviani, 1969, e da Marotti, Romei, 1991. 
consuetudine - si denegava implicitamente la possibilità che le donne calcassero le scene.

Il genere maschile degli interpreti era proprio, per esempio, dei dilettanti "ridicolosi", attori con maschera in commedia, attivi nelle occasioni carnevalesche e festive e non estranei alle tecniche dell'Arte (Mariti, 1978; Tamburini, 2012). E quando viene istituito un teatro di formazione, parte integrante dei processi pedagogici e scolastici (Majorana, 2000), i gesuiti, che di simili programmi furono i campioni, lo concepirono a loro volta esclusivamente maschile e non solo - com'è ovvio - quanto agli interpreti, allievi tutti dei loro collegi, ma anche quanto ai personaggi: nella versione definitiva, del 1599, la Ratio studiorum gesuitica regola questo punto esplicitamente (Lukács, 1986, p. 371), pur se col tempo sarà disatteso.

Giovan Domenico Ottonelli, il più prolifico argomentatore della questione del teatro comico dei professionisti italiani, gesuita anche lui, si pronunzia negativamente riguardo alla consuetudine, specialmente accademica, di «introdurre i giovani vestiti da donne nel teatro» - imbellettati, adornati, graziosamente abbigliati - in luogo di vere donne, pur quando si rappresenta «una femmina tuttoché buona sia, virtuosa e santa». E benché non saranno mai pari alle conseguenze «che nascono, come da seminario d'iniquità, dalla condotta, comparsa e conversatione delle comiche ordinarie» $(1648$, pp. 188-189, 194), gli esiti peccaminosi di tale pratica egli li considera insidiosissimi, tanto a causa della malizia, della disonestà, degli atti libidinosi che essa induce, quanto a causa dell'effettiva effeminatezza che dietro il travestimento si può nascondere. Esemplifica i rischi con un caso per più versi eloquente:

Un religioso, grave di età, persona di molta dottrina et uomo di consumata e sperimentata virtù, fu invitato, col suo principal superiore et andò a sentir un'azzione sacra, intitolata l'Invenzione della s. Croce; comparve un giovanetto con nome di s. Elena, vestito pomposamente: quel grave servo di Dio, religioso e sacerdote, non sentì punto fastidio nel tempo di recitare; ma dipoi, per molto tempo et anni, sentì grandissima e fastidiosissima pena e tentazione, quando si ricordava di quella s. Elena rappresentata. (Ibidem, pp. 394-395) 
Queste parole fanno trasparire una visione tutta morale, allusiva dell'ambiguità di genere di cui il giovanetto in vesti di donna si sarebbe fatto espressione, tanto da produrre nel vecchio sacerdote spettatore un'assillante, disordinata fantasia: la lettura di Ottonelli si ricollega appunto a ciò che presumibilmente motivava le preoccupazioni della Ratio studiorum. Egli infatti conclude che né giovani in abiti femminili, né vere donne dovrebbero calcare le scene.

È evidente che sullo sfondo dello scandalo per l'arrivo dell'attrice sulle scene c'è appunto, nella mente di chi lo solleva, la comparazione con la consuetudine della recitazione solo virile e specialmente di quella codificatasi in ambito cristiano. Il confronto fra i due teatri si stringe esemplarmente nell'Avvertimento d'uno intendente sui criteri per allestire tragedie devote, esse pure di ambiente gesuitico: uscito anonimo nel 1633 (a corredo di uno dei quattordici volumi delle importanti Tragedie sacre e morali del gesuita Ortensio Scammacca), espressione di ampia e solida competenza teatrale di orientamento cristiano, in esso si afferma che delle «due sorti di rappresentanti» di cui il teatro dispone le persone «mercenarie» non sono adatte alle tragedie, ma soltanto a quelle loro commedie che garantiscono un introito grazie a spettatori «che non cercano il profitto» spirituale, ma «il solo passatempo». All'inverso, si legge, «i rappresentatori della tragedia siano huomini gravi, che non per interesse di guadagno, ma per desiderio solo di giovare altrui si fanno vedere in scena tragica». E di seguito vi si sostiene che in questo contesto le parti di donna dovranno farle «giovenetti di buona indole, intendenti delle belle lettere et applicati agli honorati studi», meglio se membri di una «honorata academia, il cui fine sia per lo studio delle belle lettere, lo splendore della città» e l'altrui «profitto» (Avvertimento d'uno intendente, 1633, pp. 6-7). Secondo il nostro "intendente", i virtuosi cittadini cristiani hanno dunque non solo la prerogativa ma il compito di recitare opere edificanti: anche nei personaggi custodiranno l'assennatezza e la rappresentatività che sono loro proprie, trasmettendole così, santamente e civilmente, agli spettatori. Condizioni necessarie, queste, giacché, come afferma il canonico Guglielmo Baldesano, se fossero messi in scena da «persone d'ingegno vanissimo (...), di costumi perversi, (...) di vita licenziosa», questi allestimenti si appaierebbero alle «nefande co- 
medie» dei comici itineranti (Baldesano, 1969, p. 96) ${ }^{3}$, considerati corruttori per eccellenza della vita e della morale cristiane.

Nell'epoca in cui la Commedia dell'Arte si afferma, il problema del raffronto tra la recitazione di genere maschile e quella di genere femminile risulta dunque ben presente ai contemporanei. E non v'è dubbio che l'avvento dell'attrice abbia imposto un riassetto di portata straordinaria delle pratiche recitative, nel passaggio dalla interpretazione maschile alla interpretazione femminile, non meno che nell'affiancarsi di uomini e donne sulle scene professionali (Taviani, Schino, 1982, pp. 337-343) e nell'emergere inequivoco della veracità del personaggio muliebre (Mariti, 2002, p. 438): senza l'attrice la scena mancherebbe del «verisimile», dichiara il comico Giovan Battista Andreini (1625, p. 510). Mentre, dinanzi alla presenza fisica della donna recitante e all'efficacia della sua femminilità teatrale, deve essersi anche prodotta nello spettatore una mutazione del sistema di riferimento percettivo, dell'ordine psicofisico, immaginativo, intellettivo.

2. La consistenza del problema della donna in scena non può essere trascurata nemmeno in rapporto con la complessiva visione dell'attrice rispetto ad altre identità di donna.

Stati e condotte di vita femminili, nella prospettiva del disciplinamento sociale e religioso, forniscono processi di modellizzazione influenti nella società del tempo e si costituiscono come veri e propri itinerari pratici di tipo esemplare. Anche le attrici sono considerate in base a simili modelli, sia sotto il segno negativo - da parte di quanti le attaccano - sia sotto il segno positivo - da parte di quanti, all'interno delle stesse compagnie o fra gli spettatori, le lodano (Majorana, 1992; 1994, pp. 484-487; 1996).

L'elaborazione delle differenze di genere nei paesi cattolici, sottolinea Gabriella Zarri, conduce l'identità maschile a essere connotata da una funzione pubblica e sociale - e qui rientra anche la legittimità della recitazione da parte degli uomini - e l'identità femminile a definirsi in base allo status vitae: vale a dire vergine, maritata, vedova, secondo

3 Prima edizione: Roma, Zannetti, 1592. 
un'antica e confermata codificazione, nuovamente disciplinata sul piano morale, sociale e religioso. In questa tripartizione, la condizione più perfetta era considerata quella monastica, che faceva coincidere gli stati di vergine e di sposa, sposa di Cristo. Seguiva la condizione vedovile e, per ultima, quella matrimoniale, la meno perfetta, perché tale da non permettere di perseguire la contemplazione e l'unione con Dio. Questi stati di vita erano tutti e tre sottoposti al valore dell'onore, secondo un rapporto tra la società e il sesso femminile delineatosi già nel medioevo e implicante la custodia, la quale configurava di fatto uno stato di segregazione, di separatezza, tanto nei monasteri, quanto dentro il recinto domestico. Tale custodia esigeva anche tutela, un obbligo a cui provvedevano mariti, fratelli, confessori, padri spirituali, ai quali le donne dovevano obbedienza (Zarri, 2004).

L'evidenza, l'ovvietà quasi, della irriducibilità dell'attrice di mestiere al modello perfettissimo della monaca sembra non poter lasciare spazio a riflessioni. Tuttavia, tale modello è il vertice di una piramide e costituisce, come si è visto, un paradigma qualitativo inevitabile anche per le donne che vivono nello stato secolare (Schulte Van Kessel, 1991) - tutte: comprese le attrici ${ }^{4}$.

Converrà allora ragionare brevemente su questo modello, considerando quanto statuisce al riguardo il Concilio di Trento col Decretum de regularibus et monialibus del dicembre 1563 (Alberigo, Dossetti, Joannou, Leonardi, Prodi, 1973, pp. 776-784). Il decreto stabilisce fra l'altro la clausura, secondo un principio di custodia che si esprime qui al massimo grado e si riferisce particolarmente alla castità (Zarri, 1997, pp. 660-661): non è lecito che le monache escano dal monastero, in nessun caso, salvo per ragioni eccezionali e approvate dal vescovo; inoltre, a nessuno - senza distinzione di sesso e di età e sotto pena di scomuni-

4 Non risulta singolare, in questo senso, che all'interno di una famiglia dell'Arte come quella degli Andreini, accanto ai genitori e a un fratello attori di mestiere ci fossero tre o forse addirittura quattro figlie fattesi monache e un figlio monaco vallombrosano (Fiaschini, 2007, pp. 42, 94, 126-127). Similmente furono monaca agostiniana e frate domenicano anche due figli del comico Niccolò Barbieri: per l'esemplare educazione cristiana impartita loro lo loda Ottonelli, 1652, pp. 311-312. 
ca - è consentito accedere al monastero, tranne che in virtù di un'autorizzazione scritta del vescovo o del superiore.

Questo elemento del decreto pone anche a noi questioni importanti. La clausura in cui sono ristrette le monache e dalla quale la loro vita è qualificata - condizione la più perfetta per concentrare tutte sé stesse in Dio e per tenersi distinte dal mondo - si riflette anche sulle donne laiche, quale che sia il loro status. Esse pure vanno custodite in una condizione stanziale e protetta, sotto la tutela di mariti e genitori, anche quando sono madri educatrici o responsabili delle esigenze quotidiane (Solfaroli Camillocci, 1996).

Va evidenziato, allora, che all'interno del sistema teatrale italiano commerciale, feriale, nomade - tale stato non si addice alle attrici: con i loro compagni, infatti, esse viaggiano (Viaggi teatrali, 1989) e non sono pertanto condizionate dalla chiusura e dalla custodia nella dimora domestica, stabile e costante; la loro pratica di vita itinerante è socialmente palese, osservabile nello spazio, nel tempo, nei modi del viaggio. Nella vita, il loro mestiere le pone in condizioni di pubblicità, del tutto singolari rispetto a quelle delle altre donne. Benché i contratti teatrali siano per lo più sottoscritti dal padre o dal coniuge (Ferrone, 2014, p. 41), nell'ambito produttivo le attrici intrattengono rapporti paritari con i loro compagni attori; e non manifestano sottomissione nemmeno sul piano della fabula scenica, dove al contrario, le Innamorate in ispecie, mostrano la propensione a sfuggire al dominio dei padri.

La predicazione e la precettistica comportamentale prevedono inoltre che le donne, siano esse maritate o vergini, debbano a propria volta manifestare, diversamente graduato, quel segno di ritiratezza e separatezza che caratterizza la perfezione monastica. Tale condizione di riserbo deve riflettersi, assumendo caratteri riconoscibili, anche al di fuori delle mura domestiche, nelle condotte che le donne assumono nell'ambito della vita sociale. Al riguardo scrive Juan Luís Vives: «Non camini la femina né velocemente, né con troppa tardità. Sedendo tra gli huomini, stia co'l corpo e co'l volto modestamente (...). Tenga gli occhi bassi, levandoli di raro et vergognosamente; non guardi alcuno di continuo». Non stia «favoleggiante arditamente tra gli huomini per lungo spacio», con voce «sonora» o «arrogante» $(1546$, p. 92r). 
In questa definizione degli atti esteriori femminili, la modestia corrisponde allo spazio domestico protetto e operoso, appartato rispetto alla vita cittadina come agli uffici pubblici riservati all'uomo; al di fuori della casa, la privatezza si identifica con lo stare rivolte verso sé stesse, lo sguardo basso, il gesto composto, non vistoso, la voce e la parola non pervasive. Sono gli indizi coerenti della virtù femminile e, nell'attività sociale, fanno sì che le donne eludano i rapporti diretti e i contatti sensoriali e corporei con chi le circonda, tratteggiando così, in una misura più moderata, la completa clausura monastica. La modestia, si potrebbe dire, chiude le donne in sé stesse rendendole inefficaci, tali da non sperimentare e da non suscitare alcun effetto negativo, né fisico né affettivo.

A questa auspicata condotta quella delle attrici sulle scene si oppone radicalmente: basterebbe a «infettare il mondo», scrive il leonardino Cesare Franciotti, la sola «mostra sconcia» che le comiche danno di sé sulla scena, «quando a bello studio, con artifitio histrionico», alle parole e ai canti uniscono «i movimenti della persona, gli sguardi, i sospiri, gli sdegni, (...) gli abbracciamenti et altro di peggiore» (1611, pp. 339-340). Al confronto con la modestia che deve essere propria delle une, le altre dunque - le attrici - sono connotate da appariscenza espressiva e relazionale, da sicurezza di sé, dall'essere attive nell'intraprendere con efficace competenza iniziative che coinvolgono lo spettro più esteso del linguaggio verbale e corporeo; sono eccessive, proprio nel senso dell'eccedere la norma, vistose.

Se il paradigma claustrale esige che il monastero sia un luogo inviolabile, invalicabile, il teatro, all'inverso, è un luogo diretto, aperto, comunicante: ma in quale modo comunica? La scena si sottrae per convenzione a qualunque contaminazione: coloro che vi si accostano da spettatori, accettando la finzione, non possono varcare a discrezione il confine ideale o fisico che li separa dal luogo dell'azione. Il teatro non è, dunque, uno spazio disponibile al contatto fisico tra attori e pubblico, bensì, uno spazio caratterizzato dalla loro reciproca distanza. Ciò che colma tale distanza, tuttavia, è lo sguardo il quale, proprio grazie alla invenzione del teatro - scrive Derrick de Kerckhove - è diventato «l'organo più adatto a "contenere" e integrare le percezioni offerte dagli altri sensi» (1995, p. 89). La legge che regola l'assoluta clausura monastica 
segnala invece il grado zero della reciprocità visiva fra chi appartiene al chiostro e chi ne è fuori, cosicché lo sguardo sfrontato, nelle monache, era specialmente riprovato e doveva essere prontamente emendato o altrimenti punito rigorosamente (Bianchini, 1996, p. 203).

Anche tale norma fonda una progressione: nello stato laicale la donna dovrà condursi, lo abbiamo visto, con gli occhi bassi (Pozzi, 1986; Zarri, 1994, pp. 257-258), non dovrà guardarsi dattorno o, peggio, suscitare sguardi su di sé: giacché con gli occhi ella esercita il potere distruttivo dell'indurre il peccato, essendo per sua natura - scrive il gesuita Paolo Segneri - «un'immagine viva, sustanziale e spirante», che «a bello studio, co' guardi, co' cenni, col colore, con l'abito scandaloso» può fare «pubblica strage di chi la guarda» (1686, p. 451). La donna virtuosa appartiene solo allo sguardo di Dio e a quello del marito, mentre provoca desideri lussuriosi nel momento in cui si offre alla vista di molti (Passi, 1602, p. 165). Al contrario, la vera «sconfitta delle cristiane perfezzioni» viene appunto dalla vista della «comica di professione, perita dell'arte, pratica della scena, formosa per natura, speciosa per artificio et ornata con pompa e con vanissima diligenza», scrive fra gli altri Ottonelli ${ }^{5}$. E conclude: «Io noto ch'una sola occhiata basta qualche volta per rapir il cuore e l'affetto d'uno spettatore» (1648, pp. 131-132).

L'attrice è dunque il campione del guardare, del guardare senza condizioni: non soltanto perché, sulla scena, attraverso la necessaria reciprocità dello sguardo fra comici, stabilisce con gli uomini relazioni anche drammaturgicamente rilevanti; ma soprattutto perché a un tempo riceve dal pubblico gli sguardi di tanti e li deposita essa stessa su di loro, facendo proprio un costume legittimo, invece, soltanto per l'uomo. Guarda apertamente gli spettatori e si esibisce a beneficio del loro sguardo: ella fonda il proprio potere sugli occhi, strumento di un contatto favorito dalle azioni inscenate. Attrice in scena e spettatore dinanzi a lei non possono toccarsi, ma si guardano; uno scambio, questo loro, considerato pari a quello carnale.

5 Le lodi in onore delle comiche dell'Arte, scritte da attori e da spettatori, fanno riferimento appunto alla sapienza tecnica che distingue le attrici dalle donne comuni (Taviani, 1986, pp. 64-73; Marotti, Romei, 1991). 
3. Il rimando dei moralisti alla prostituta, quando parlano dell'attrice, è infatti frequente (Tessari, 1989). E nel 1646 esce un ponderoso volume - che mi pare non sia mai stato preso in considerazione negli studi sull'attrice dell'Arte - nel quale questo legame viene tematizzato in modo sistematico. Si tratta Della pericolosa conversatione con le donne, o poco modeste o ritirate o cantatrici o accademiche e si deve ancora una volta a Giovan Domenico Ottonelli'. L'autore, com'è noto, ha ampia cognizione della scena dell'Arte, di cui tratta nei sei tomi Della christiana moderatione del theatro (1646-1652), anche più sopra citati.

La "pericolosa conversazione" di cui egli parla è quella vana e inutile, la quale procura danno all'anima del cristiano e lo spinge verso il pericolo infernale. Si contrappone alla "cristiana conversazione", adeguamento controriformistico della "civil conversazione" cortigiana: strumento della educazione laica, è «quella che mira ad insegnare i princìpi della dottrina cattolica, le forme della devozione, i modelli del vivere da vero e buon cristiano» (Casali, 1996, p. 300).

Quello di Ottonelli sulla pericolosa conversazione è un trattato di quasi seicento pagine, d'impianto teorico-morale, diviso in trenta punti. In almeno venti di questi, l'oggetto esplicito della riflessione è appunto l'attrice (benché il titolo non vi alluda affatto), quella che l'autore vi definisce «comica impudica», sempre associandola alla «publica meretrice». Egli non annovera fra quelle donne che genericamente indica come «triste» nessun'altra categoria femminile: soltanto l'attrice e la prostituta. Nella prospettiva di Ottonelli siamo lontani dalla ipotesi avanzata a suo tempo da Taviani e Schino che possa essersi verificato un avvicendamento tra le meretrices honestae della metà del XVI secolo e le comiche di professione affermatesi di lì in avanti; le quali, di quelle cortigiane (donne coltivate, dedite all'intrattenimento poetico, musicale e canoro, alla conversazione, appunto, alle lettere, e che le loro arti raffinate, offerte negli ambienti scelti della città, avevano reso tanto attraenti

6 Già edito con titolo diverso nel 1645 (Risposta al quesito che male sia andare a conversatione in casa di una persona poco modesta), nello stesso 1646 il testo viene ripreso dall'autore anche in altra opera simile (Alcuni buoni avvisi e casi di coscienza intorno alla pericolosa conversatione, da proporsi a chi conversa poco modestamente). 
e fatto oggetto di ammirazione ed encomi), avrebbero assunto il ruolo sociale, le proprietà seduttive, maturando poi sulle scene parallele abilità (Taviani, Schino, 1988, pp. 335-337).

Attrici che si concedevano sessualmente in cambio di danaro ce n'erano (Ferrone, 2014, p. 41); tuttavia è proprio riguardo alla conversazione che l'assimilazione della meretrice alla comica proposta da Ottonelli risulta singolare: mentre l'una, infatti, vive in modo stanziale e domestico la propria attività e quindi riceve in casa, tanto per conversare quanto per vendere la propria prestazione sessuale (Lawner, 1988; Cohen, 1991), l'altra, al contrario, conducendo vita nomade, sta raramente fissa in un alloggio e svolge la propria attività professionale nei teatri, cioè non in un luogo privato, ma accessibile a pagamento a un'udienza indifferenziata, di ogni età, sesso e condizione, che vi si reca in primo luogo per assistere alla rappresentazione e non per stabilire rapporti diretti con gli interpreti.

Quando nel suo Della pericolosa conversatione Ottonelli entra nel dettaglio del mestiere delle attrici non dice che oltre alla professione scenica esse vendono anche il corpo. Non parla di loro né come di prostitute né come di conversatrici private, ma come di recitanti; e in quanto tali, non perché usano la parola con arte - come sarebbe logico nel quadro appunto della conversazione - ma proprio perché si esibiscono pubblicamente in scena. Afferma, per esempio, che l'inclinazione al vizio «si fa maggiore in un huomo di poco spirito, che pecca con maggior facilità conversando in casa, che rimirando da lontano nel theatro». Oppure: «la mala conversatione (...) è quella che si fa $(. .$.$) in casa di femmina per-$ duta nel meretrico o nella theatrale oscenità e per[ci] ò è conversatione scandalosa». E retoricamente si interroga su «Qual sia cosa peggiore di queste due: l'andar a conversatione in casa di una donna impudica, overo andar a sentire in theatro una oscena rappresentatione» (1646, pp. 297, 337, 378).

Che cosa testimonia allora, Ottonelli, nel dare per scontato senza argomentarlo che quei due generi di donna, e solo quelli, accomunati dalla pratica della conversazione, rappresentino pericolo mortale per l'anima degli uomini "conversanti"? Perché, in questo contesto, le assimila? Il punto è, mi pare, che a quest'epoca proprio la meretrice e la 
comica dell'Arte sono le donne pubbliche per eccellenza. Le sole, se si trascurano quelle che infatti lo stesso Ottonelli richiama nel trattato, riconoscendo loro, però, molte virtù: e cioè la esigua schiera di accademiche, donne coltivate che tengono discorsi al cospetto di un pubblico. Con queste si corre solo «qualche leggier pericolo di peccato» a causa del «duplicato atto» degli uditori, quello «di udire una donna parlante con dottrina, con arte e con affetto, publicamente; e [quello] di mirar una donna con qualche lunghezza di tempo e con molta applicatione di senso» (Ibidem, pp. 404-405, 406-439).

La visione dell'attrice dell'Arte qui proposta da Ottonelli spinge dunque, senza esitazioni, l'identità e il sentimento sociale che si avrebbe di lei verso la prostituta: entrambe dotate di specifiche competenze e abilità, a fini economici sfruttano il loro aspetto, l'insolenza dello sguardo, la provocazione del gesto, la sconvenienza dell'abito, l'uso dei belletti, la capacità di intrattenere e svagare, la vita pubblica, la facilità di relazione con gli uomini, l'assenza di custodia e di tutela. Si collocano dunque all'estremo opposto di quella scala alla base della quale abbiamo trovato il fondamento monastico dei modelli femminili.

Fra le donne cristiane del tempo appartenenti agli stati di vita regolari e regolati - monache, vedove e maritate, appunto - ce ne erano nondimeno di insubordinate alle norme previste, capaci di manifestare comportamenti socialmente e spiritualmente alieni dagli schemi di vita promossi e accettati (Bianchini, 1996): l'auspicata disciplina sociale e religiosa era dunque anche violata. Queste donne, però, sono peccatrici individue, non costituiscono al pari delle attrici (e delle prostitute) una categoria unitaria. Le comiche dell'Arte, nella loro condizione di professioniste della scena, formano invece un insieme preciso e omogeneo, un insieme riconoscibile per la comune estetica recitativa e le condotte di vita condivise. Inoltre si costituiscono come un gruppo uniforme anche per essere ritenute estranee alla codificazione dei modelli civili e cristiani. Nel loro insieme - come abbiamo visto - sono decisamente intese come un anti-modello. In questo senso, anche rispetto ad altre donne individualmente trasgressive, la presenza delle attrici è sentita a maggior ragione come una rottura nella cultura dell'ordine e del disci- 
plinamento sociale e morale e, più in particolare, delle coordinate della identità femminile nell'Italia cattolica di età moderna.

L'identità delle attrici viene dunque collocata al di fuori della mappa sociale della perfezione cristiana; ma ciò non significa che la loro identità non si venga a formare nel rapporto dialettico con i modelli di virtù femminile condivisi in sede morale e teologica. Risponde a questa possibile dialettica, non per nulla, proprio quanto i sostenitori delle attrici affermano, come nel caso celebre del ritratto della madre Isabella delineato da Giovan Battista Andreini ne La ferza (1625, pp. 34-35), che va considerato il risultato esemplare, criticamente ottenuto, dell'accordo tra la figura d'attrice e i modelli ideali di donna cristiana.

\section{BIBLIOGRAFIA}

Alberigo, G., Dossetti, G.L., Joannou, P.-P., Leonardi, C., \& Prodi, P. (Eds.). (1973). Conciliorum oecomenicorum decreta (2 ${ }^{\mathrm{a}}$ ed.). Bologna: Dehoniane.

Andreini, G.B. (1625). La ferza. Ragionamento secondo contra l'accuse date alla commedia. Parigi: Nicolao Callemont.

Avvertimento d'uno intendente (1633). Avvertimento d'uno intendente intorno al modo di rappresentar queste tragedie e tutte l'altre che sono per comporsi regolatamente. In O. Scammacca, Delle tragedie sacre e morali (vol. 5, pp. 5-20). Palermo.

Baldesano, G. (1969). Stimolo alle virtù del giovane christiano. In F. Taviani, La commedia dell'arte e la società barocca. La fascinazione del teatro (pp. 96-106: Del fuggire li teatri e le vanità de gli spettacoli). Roma: Bulzoni.

Bianchini, F. (1996). Regola del vivere, regola del convivere. In G. Zarri (Ed.), Donna, disciplina e creanza cristiana dal XV al XVII secolo. Studi e testi a stampa (pp. 189-204). Roma: Edizioni di storia e letteratura.

Bucci, E., \& Sgrosso, M. (2004). La pazzia di Isabella. Vita e morte di comici Gelosi. Drammaturgia dello spettacolo. Culture teatrali, 10 (a cura di G. Guccini, L'arte dei comici. Omaggio a Isabella Andreini nel quarto centenario della morte (1604-2004)), 171-187.

Casali, E. (1996). Il monastero. La villa. La canonica. Aspetti della «cristiana conversazione» nella letteratura precettistica ravennate del secondo Cinquecento. In G. Zarri (Ed.), Donna, disciplina, creanza cristiana dal 
XV al XVII secolo. Studi e testi a stampa (pp. 285-300). Roma: Edizioni di storia e letteratura.

Ciancarelli, R. (2008). Sistemi teatrali nel seicento. Strategie di comici e dilettanti nel teatro italiano del XVII secolo. Roma: Bulzoni.

Cohen, E.S. (1991). Camilla la Magra, prostituta romana. In O. Niccoli (Ed.), Rinascimento al femminile (pp. 163-196). Roma-Bari: Laterza.

De Kerckhove, D. (1995). La civilizzazione video-cristiana, Milano: Feltrinelli.

Ferrone, S. (2014). La Commedia dell'Arte. Attrici e attori italiani in Europa (XVI-XVIII secolo). Torino: Einaudi.

Fiaschini, F. (2007). L'«incessabil agitazione». Giovan Battista Andreini tra professione teatrale, cultura letteraria e religione. Pisa: Giardini.

Franciotti, C. (1611). Il giovane christiano [...]. Nel quale si tratta in che modo si devino instruire i buoni giovani alla devotione, per vivere in gratia di Dio. Venezia: Bernardo Giunti.

Lawner, L. (1988). Le cortigiane. Ritratti del Rinascimento. Milano: Rizzoli.

Lukács, L. (Ed.). (1986). Ratio atque institutio studiorum Societatis Iesu (1586, 1591, 1599). Roma: Institutum Historicum Societatis Iesu (Monumenta historica Societatis Iesu, 129).

Majorana, B. (1992). «Un gemino valor»: mestiere e virtù dei comici dell’Arte nel primo Seicento. Medioevo e Rinascimento, 6 n.s. (3), 173-193.

Majorana, B. (1994). Governo del corpo, governo dell'anima: attori e spettatori nel teatro italiano del XVII secolo. In P. Prodi (Ed.), Disciplina dell'anima, disciplina del corpo e disciplina della società tra medio evo ed età moderna (pp. 437-490). Bologna: Il Mulino.

Majorana, B. (1996). Finzioni, imitazioni, azioni: donne e teatro. In G. Zarri (Ed.), Donna, disciplina, creanza cristiana dal XV al XVII secolo. Studi e testi a stampa (pp. 121-139). Roma: Edizioni di storia e letteratura.

Majorana, B. (2000). La scena dell'eloquenza. In R. Alonge \& G. Davico Bonino (Eds.), Storia del teatro moderno e contemporaneo, vol. 1: La nascita del teatro moderno. Cinquecento e seicento (pp. 1043-1066). Torino: Einaudi.

Majorana, B. (2013). L'attore necessario: drammaturgia della santità e santità rappresentata. In T. Lewicki (Ed.), L'eroe sensibile: evoluzione del teatro agiografico nel primo '600. Atti del Convegno Internazionale (Roma, 29-31 ottobre 2009) (pp. 69-107). Varsavia: Wydawnictwo Salezjańskie. 
Mariti, L. (1978). Commedia ridicolosa. Comici di professione, dilettanti, editoria teatrale nel Seicento. Storia e testi. Roma: Bulzoni.

Mariti, L. (2002). Valore e coscienza del teatro in età barocca. In I capricci di Proteo. Percorsi e linguaggi del barocco. Atti del Convegno Internazionale (Lecce, 23-26 ottobre 2000) (pp. 419-455). Roma: Salerno.

Marotti, F., \& Romei, G. (1991). La commedia dell'arte e la società barocca. La professione del teatro. Roma: Bulzoni.

Mazzoni, S. (2000). Lo spettacolo delle accademie. In R. Alonge \& G. Davico Bonino (Eds.), Storia del teatro moderno e contemporaneo, vol. 1: La nascita del teatro moderno. Cinquecento e seicento (pp. 869-904). Torino: Einaudi.

Ottonelli, G.D. (1646). Della pericolosa conversatione con le donne, o poco modeste, o ritirate, o cantatrici, o accademiche. Firenze: Luca Franceschini \& Alessandro Logi.

Ottonelli, G.D. (1648). Della christiana moderatione del theatro, vol. 1 ( $2^{\mathrm{a}}$ ed.). Firenze: Luca Franceschini \& Alessandro Logi.

Ottonelli, G.D. (1652). Della christiana moderatione del theatro, vol. 4. Firenze: Bonardi.

Passi, G. (1602). Dello stato maritale. Venezia: Iacomo Antonio Somascho.

Pozzi, G. (1986). Occhi bassi. In E. Marsch \& G. Pozzi (Eds.), Thematologie des Kleinen (pp. 161-211). Fribourg: Éditions Universitaires.

Schulte Van Kessel, E. (1991). Vergini e madri tra cielo e terra. Le cristiane nella prima età moderna. In N. Zemon Davis \& A. Farge, Storia delle donne dal Rinascimento all'età moderna (pp. 156-200). Roma-Bari: Laterza.

Segneri, P. (1686). Il cristiano instruito nella sua legge. Ragionamenti morali. Firenze: Stamperia di S.A.S.

Solfaroli Camillocci, D. (1996). L'obbedienza femminile tra virtù domestiche e disciplina monastica. In G. Zarri (Ed.), Donna, disciplina e creanza cristiana dal XV al XVII secolo. Studi e testi a stampa (pp. 269-283). Roma: Edizioni di storia e letteratura.

Tamburini, E. (2012). Gian Lorenzo Bernini e il teatro dell'arte. Firenze: Le lettere.

Taviani, F. (1969). La commedia dell'arte e la società barocca. La fascinazione del teatro. Roma: Bulzoni.

Taviani, F. (1986). Un vivo contrasto. Seminario su attrici e attori della commedia dell'arte. Teatro e storia, 1(1), 25-75. 
Taviani, F., \& Schino M. (1982). Il segreto della commedia dell'arte. La memoria delle compagnie italiane del XVI, XVII e XVIII secolo. Firenze: La casa Usher.

Viaggi teatrali dall'Italia a Parigi fra Cinque e Seicento, Atti del Convegno Internazionale (Torino, 6-8 aprile 1987). (1989). Genova: Costa \& Nolan.

Vives, G.L. (1546). De l'ufficio del marito, come si debba portare verso la moglie. De l'istitutione de la femina christiana, vergine, maritata o vedova. De lo ammaestrare i fanciulli ne le arti liberali. Venezia: Vincenzo Vaugris.

Zarri, G. (1994). Disciplina regolare e pratica di coscienza: le virtù e i comportamenti sociali in comunità femminili (secc. XVI-XVIII). In P. Prodi (Ed.), Disciplina dell'anima, disciplina del corpo e disciplina della società tra medioevo ed età moderna (pp. 257-278). Bologna: il Mulino.

Zarri, G. (1997). Monasteri femminili in Italia nel secolo XVI. Rivista di storia e letteratura religiosa, 33(3), 643-669.

Zarri, G. (2004). Disciplina tridentina. L'istituzione dell'identità femminile nell'Italia moderna. In M. Borsari \& D. Francesconi (Eds.), Maschio e femmina li creò. L'elaborazione religiosa delle differenze di genere (pp. 135-171). Modena: Banca Popolare dell'Emilia Romagna.

\footnotetext{
Riassunto: Il mestiere di comica dell'Arte assume una posizione marcatamente visibile nell'orizzonte pubblico dell'Italia cattolica di età moderna, dove l'attrice si colloca tanto sui confini delle pratiche teatrali maschili edificanti dei dilettanti quanto su quelli dei modelli femminili virtuosi (monache, spose, vergini), confliggendo con gli uni e con gli altri. Queste contrapposizioni dovevano essere ben presenti in primo luogo agli spettatori, i quali potevano operare un confronto sia con spettacoli non professionistici e dunque recitati soltanto da uomini, sia con modelli diversi di donna. I contrasti vengono indagati attraverso la trattatistica morale del tempo, soffermandosi infine sull'assimilazione fra attrice e prostituta proposta in un corposo trattato del 1646 di G.D. Ottonelli.
}

Parole chiave: Commedia dell'Arte, attrici, attori dilettanti, modelli femminili, prostituta 